\title{
Autologous Platelet Rich Plasma (PRP) - Regeneration to Repair in Post Operated Caesarean Wounds
}

\author{
Jyotsana Abhay Gawri1, Deepti Sandeep Shrivastav², Manjusha Agrawal ${ }^{3}$, Syed Shabnam Jahan ${ }^{4}$ \\ 1, 2, 3, 4 Department of Obstetrics and Gynaecology, JNMC, DMIMS, Wardha, Maharashtra, India.
}

\section{INTRODUCTION}

Autologous Platelet-Rich Plasma (PRP) therapy is a treatment based on high concentrations of growth factors released by platelets, having potential to improve the healing by stimulating regeneration of epithelium. In this report, we describe three patients with non-healing wounds after major surgeries (post caesarean) at our institution who were efficiently \& contentedly treated with PRP with positive results. Also we reviewed the literature of PRP's mechanisms, benefits \& limitations.

Wound healing is an intricate process characterized by stages of inflammation, proliferation, repair, and remodelling. ${ }^{(1,2)}$ The stage of inflammation is commenced partly by platelet degranulation.(1) Platelets have a minimum of five growth factors which will lead to tissue development and epithelialization: Platelet factor 4, PlateletDerived Growth Factor (PDGF), platelet-derived angiogenesis factor, transforming growth factors and platelet derived epidermal growth factor. These factors are important in wound healing because they stimulate and modulate multiple biological processes on their release. $(1,2,3,4)$ Chronic wounds may lack growth factors in some cases.(2) Reduced growth factor availability may be due to reduced production, reduced release, excess degradation, trapping or a combination of these processes. ${ }^{(2)}$

Caesarean delivery rate has continued to increase \& caesarean deliveries are being employed for about $15 \%$ of the births worldwide. (5) Surgical site problems like infection, haematoma, dehiscence, pain \& seroma can occur within the postpartum period. The postpartum period may be challenging time \& difficult for mothers especially due to the needs of caring for a new-born baby, these complications are related to prolonged hospital stay, increased cost \& substantial morbidity and mortality. Maternal and neonatal care can be improved by reducing the extent of these morbidities, therefore scaling down on medical expenses. ${ }^{(6)}$ The risk factors affecting the wound repair process in caesarean delivery includes: (1) chronic systemic illness (diabetes, hypertension, and immune deficiencies), (2) Anaemia, (3) Time Span of Surgery > 90 minutes, (4) Obesity, (5) Previous Incision, (6) Corticosteroid Treatment, (7) Immunosuppression Treatment, and (8) Twin Births. $(7,8)$

PRP is an autologous product extracted from whole blood there by concentrating a large number of platelets in a small proportion of plasma through the process of gradient density centrifugation. Platelets play major role in the process of haemostasis and later wound healing in any wound. Platelets initially get accumulated and form plug producing haemostasis during the process of injury. Platelet membrane gets depolarised by the action of thrombin \& release platelet granules which are rich in various growth factors like PDGF (Platelet-Derived Growth Factor), FGF (Fibroblast Growth Factor), and interleukins. $(9,10)$ These growth factors aid in the process of wound healing by laying collagen matrix, fibroblast proliferation and early maturation of collagen. Based on this background, autologous platelet growth factors in the form of plasma rich platelet started in the management of chronic wounds.
Corresponding Author: Dr. Deepti Sandeep Shrivastav, AVBRH, DMIMS, Sawangi, Wardha, Maharashtra, India. E-mail: deepti_shrivastav69@yahoo.com

DOI: $10.14260 / \mathrm{jemds} / 2020 / 716$

How to Cite This Article:

Gawri JA, Shrivastav DS, Agrawal M, et al. Autologous platelet rich plasma (PRP)regeneration to repair in post operated caesarean wounds. J. Evolution Med. Dent. Sci. 2020;9(43):3261-3264, DOI: $10.14260 / j e m d s / 2020 / 716$

Submission 18-02-2020,

Peer Review 06-05-2020

Acceptance 13-05-2020,

Published 26-10-2020.

Copyright (C) 2020 JEMDS. This is an open access article distributed under Creative Commons Attribution License [Attribution 4.0 International (CC BY 4.0)] 


\section{PREPARATION OF PRP \& APPLICATION}

After explaining the treatment protocol and other options, informed valid written consent was taken from all patients. Autologous PRP was prepared by centrifugation of the patient's blood for $15 \mathrm{~min}$ at $3500 \mathrm{rpm}$ in a tabletop centrifuge after aseptic collection from the ante cubital vein employing a $16 \mathrm{G}$ needle, which is then transferred to a sterile vacutainer. Anti-coagulant was not used in this study. After fractionation of components the middle buffy layer of PRP was collected; applied over wound \& dressed in sterile Vaseline gauze. The first look dressing is on 5th day which is then followed by alternate saline dressing. Based on the response of wound to the first procedure / application decision is taken to repeat the procedure of subsequent applications. As autologous platelets are used there was no chance of spread of infectious diseases and allergic reactions. The wound was followed up under the following parameters like size of wound [with tape], quality of granulation tissue, no of times of application, skin grafting, and time taken for complete healing.

\section{RESULTS}

\section{Case 1}
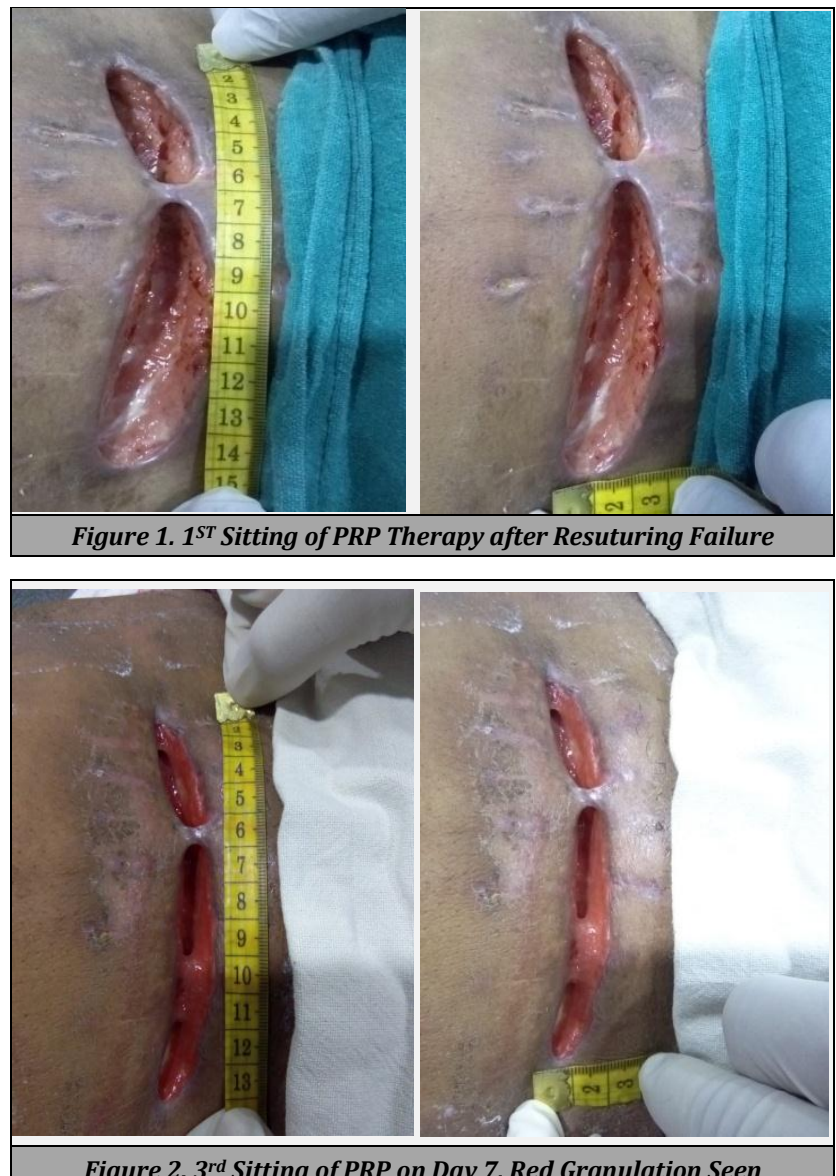

Figure 2. $3^{\text {rd }}$ Sitting of PRP on Day 7, Red Granulation Seen

Patient 1 was a 29 yrs. G2A1 with 37 weeks 4 days of gestation who had caesarean in May 2019 at our institution with indication of cephalopelvic disproportion in labour. Postoperatively she had indurated suture site, later on had infection at suture site \& fluid collection in subcutaneous planes (on ultrasonography), she was taken up for incision \& drainage. After the infection was treated with Inj. Amikacin $500 \mathrm{mg}$ bd for 7 days \& debridement of necrotic tissue, patient was taken for resuturing \& wound did not heal after resuturing. After resuturing failure, patient undertook complete 6 PRP treatments over 6 weeks. The wound became smaller \& gradually healed completely with time with subsequent PRP treatments.

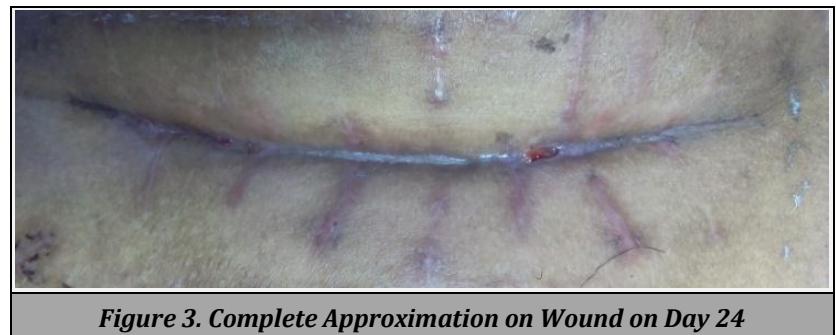

Figure 3. Complete Approximation on Wound on Day 24

\section{Case 2}
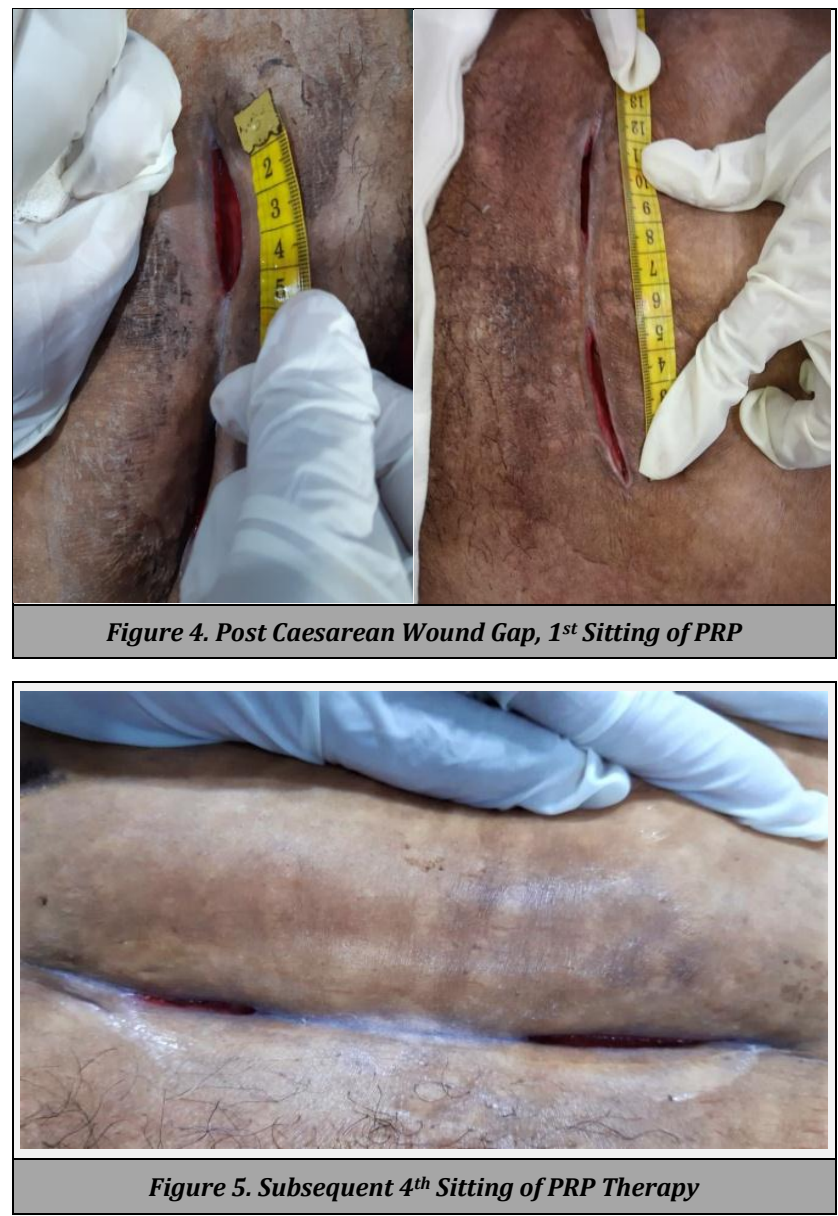

Patient 2 was a 31 yrs. old female who underwent caesarean in July, 2019 \& presented on day 15 post caesarean with complaints of pain over suture site \& wound discharge. After investigations she was found to be anaemic with haemoglobin of $8 \mathrm{gm} \mathrm{\%}$. Ultrasonography of local site over suture line was done suggestive of gross oedema \& collection with thick echoes within along the suture line in subcutaneous plane. Wound swab report was suggestive of clindamycin resistant MRSA (Methicillin-Resistant Staphylococcus aureus) coagulase positive staphylococci sensitive to amikacin. Treatment with 
antibiotics completed \& wound debridement was done. In place of resuturing, PRP treatment was introduced after anaemia correction \& wound improved gradually after 5 PRP treatments.

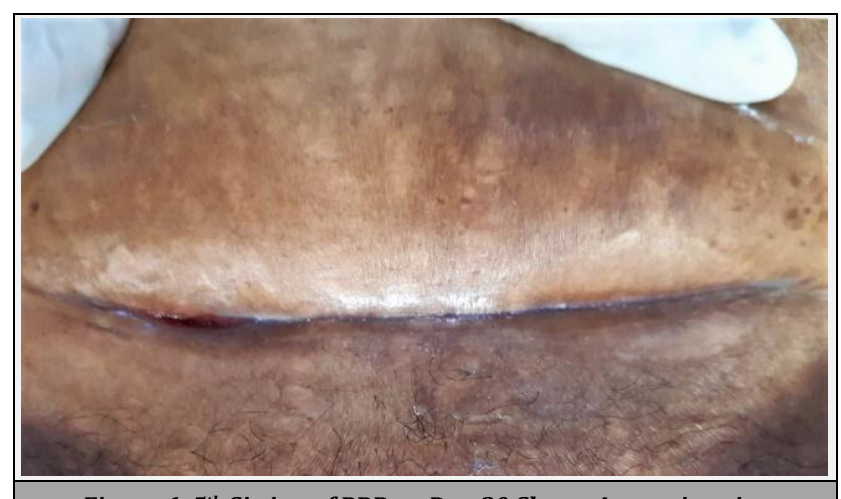

Figure 6. $5^{\text {th }}$ Sitting of PRP on Day 30 Shows Approximation

\section{Case 3}

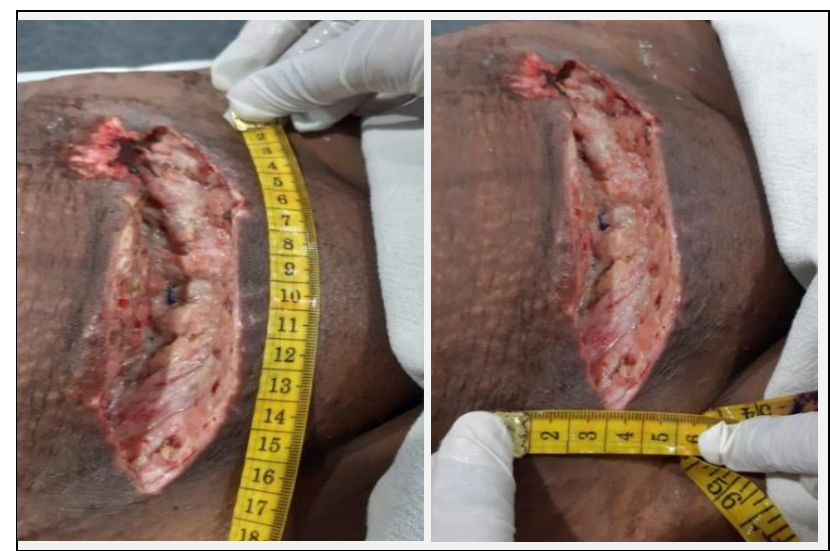

Figure 7. Wound Gap Post Caesarean, $1^{\text {st }}$ Sitting of PRP Therapy

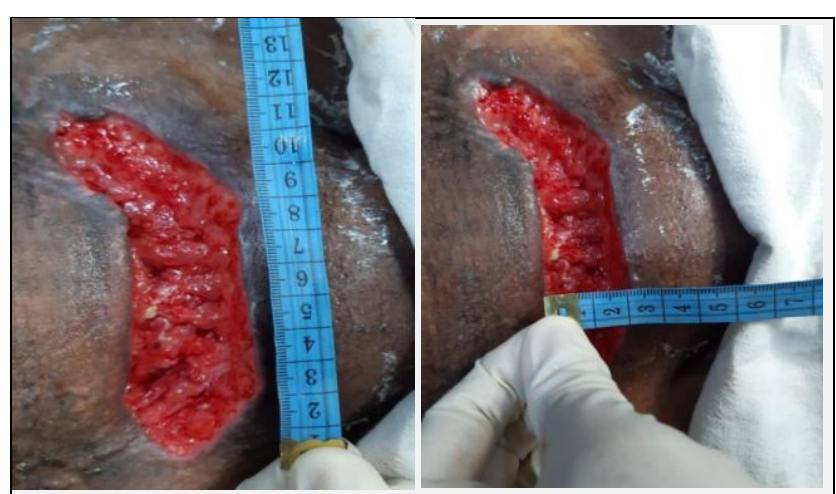

Figure 8. Healthy Granulation Seen, Subsequent $4^{\text {th }}$ Sitting of PRP on Day 16

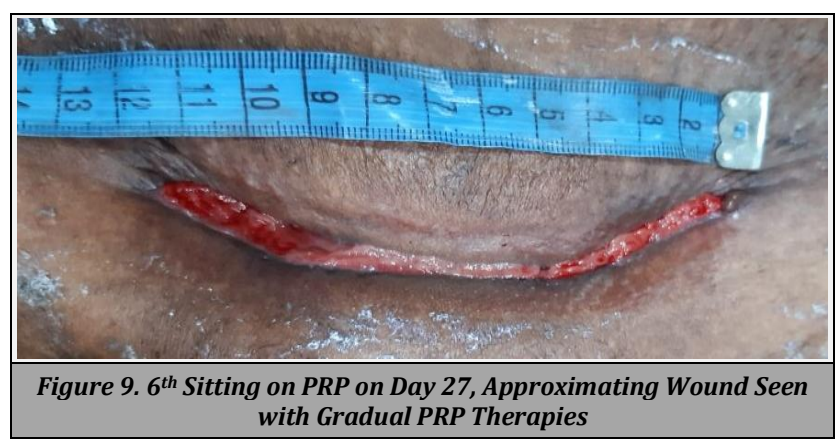

Patient 3, was a 20 yrs. primigravida with 40 weeks of gestation who came to our hospital in September 2019 \& underwent caesarean in view of cephalopelvic disproportion $\&$ had wound discharge in postoperative period. Proper antibiotic coverage was given according to wound swab report with inj Amikacin $500 \mathrm{mg}$ bd for 7 days. Despite proper daily dressing the wound did not show any improvement \& patient had wound gap. Therefore, we decided to introduce PRP treatment \& total of 13 PRP sessions were given resulting in wound healing. The wound gap was gradually filled with red granulation tissue with the wound very close to being epithelialized with gradual PRP treatment.

\section{DISCUSSION}

Chronic non-healing wounds represent a forbidding challenge and need specialized therapy to give the best advantage to each patient for wound closure. Delayed wound healing is a result of interplay of various factors. Strong association is seen between physical factors like diabetes, physical pressure, aging, vasculitis syndrome \& delayed healing of wound. Also, local influences like bacterial colonization, high levels of inflammatory cytokines, low density of beneficial cytokines \& imbalance in matrix metalloproteinase also play a role in delayed wound healing.(11,12) These factors create a vicious circle for the wound to heal, thus are the reason \& consequence of delayed wound repair. Therefore, it's essential to treat both physical \& regional influences that cause delayed wound repair. Now a variety of novel therapies are accessible to support wound healing including growth factor products, hyperbaric oxygen therapy.(13) Amongst those promising therapies is autologous platelet concentrated plasma. In this case series, we have enumerated longstanding bad wounds in post caesarean patients which fruitfully healed with PRP.

Blood platelets also play role in promoting wound healing. Platelets have abundant cytokines \& mend tissue injury by neoangiogenesis \& stimulating multiplication, immigration \& differentiation of dermal fibroblasts. $(14,15,16)$ Alpha-granules within platelets attach with platelet cytoplasmic membrane \& liberate it's constituents into the surrounding after tissue injury. Principal cytokines like VEGF (Vascular Endothelial Growth Factor), PDGF (Platelet-Derived Growth Factor) , TGFBeta (Transforming Growth Factor), epidermal growth factor \& basic fibroblast growth factor are liberated during wound healing. These growth factors not just trigger the targeted cells, but further team up with one another \& boost their functions. There are two other important roles of platelet in wound repair, first they function as a meshwork of fibrin that forms a biological scaffold. The scaffold helps mesenchymal cells to immigrate from the base \& margins of the wound, finally evolving into granulation tissue. Second they help to reduce bacterial colonization as they have antibacterial proteins. ${ }^{(17)}$

PRP therapy is easy to apply, painless \& more tolerated in patients.(18) One more advantage of PRP is safety. As an autologous preparation, PRP is safer to use than allogenic or homologous preparations \& is free from concerns over transmissible diseases like hepatitis, human immunodeficiency virus, West Nile Fever \& Creutzfeldt Jacob Disease. The risk of graft vs host disease is prevented,(19) as PRP requires no specific considerations regarding antibody 
formation \& leading to better allowance by patients. As only small volume of blood is taken from the patient each time, PRP is minimally invasive \& relatively economic modality that requires multiple venesections. Each preparation of PRP should have a minimum of 1 million platelets per microlitre.

Nevertheless, there are certain restraints regarding PRP treatment. The foremost limitation could be that hardly a small count of doctors are presently able to utilize PRP therapy in their practice due to lack of uniform protocol \& limitation of facilities. (20) Also, there can be variation in the characteristic of autologous PRP in comparison to allogenic or homologous products drawn from healthy patient's blood following systematized working methods of blood establishments. In certain patients, especially those with diabetes, PRP therapy may not be as efficacious as expected as viability \& proportion of cytokines in platelets are reported to decline.(13)

\section{CONCLUSIONS}

Platelet rich concentrate supplements deficient \& faulty wound environment by innately balancing growth factors in wound. PRP helps to restore the natural healing process. If further evidence on its productiveness is gathered \& a concrete protocol \& policy is established, PRP treatment may become one the most definitive treatments.

Financial or other competing interests: None.

Disclosure forms provided by the authors are available with the full text of this article at jemds.com

\section{REFERENCES}

[1] Bennett NT, Schultz GS. Growth factors and wound healing: Part II. Role in normal and chronic wound healing. Am J Surg 1993;166(1):74-81.

[2] Crovetti G, Martinelli G, Issi M, et al. Platelet gel for healing cutaneous chronic wounds. Transfus Apheresis Sci 2004;30(2):145-51.

[3] Weed B, Davis MDP, Felty CL, et al. Autologous platelet lysate product versus placebo in patients with chronic leg ulcerations: A pilot study using a randomized, doubleblind, placebo-controlled trial. Wounds 2004;16(9):27282.

[4] Herouy Y, Mellios P, Bandemir E, et al. Autologous platelet-derived wound healing factor promotes angiogenesis via alphavbeta3-integrin expression in chronic wounds. Int J Mol Med 2000;6(5):515-9.

[5] Betran AP, Merialdi M, Lauer JA, et al. Rates of caesarean section: analysis of global, regional and national estimates. Paediatr Perinat Epidemiol 2007;21(2):98113.
[6] Fanning J, Murrain L, Flora R, et al. Phase I/II prospective trial of autologous platelet tissue graft in gynecologic surgery. J Minim Invasive Gynecol 2007;14(5):633-7.

[7] Andrews WW, Hauth JC, Cliver SP, et al. Randomized clinical trial of extended spectrum antibiotic prophylaxis with coverage for Ureaplasma urealyticum to reduce post-cesarean delivery endometritis. Obstet Gynecol 2003;101(6):1183-9.

[8] Chaim W, Bashiri A, Bar-David J, et al. Prevalence and clinical significance of postpartum endometritis and wound infection. Infect Dis Obstet Gynecol 2000;8(2):7782.

[9] Kahle B, Hermanns HJ, Gallenkemper G. Evidence-based treatment of chronic leg ulcers. Deutsches Arzteblatt International 2011;108(14):231-7.

[10] Rayner R, Carville K, Keaton J, et al. Leg ulcers: atypical presentations and associated co morbidities. Wound Practice and Research 2009;17(4):168-85.

[11] Rappl LM. Effect of platelet rich plasma gel in a physiologically relevant platelet concentration on wounds in persons with spinal cord injury. Int Wound J 2011;8(2):187-95.

[12] Cooper DM, Yu EZ, Hennessey P, et al. Determination of endogenous cytokines in chronic wounds. Ann Surg 1994;219(6):688-91; discussion 691-2.

[13] Baltzis D, Eleftheriadou I, Veves A. Pathogenesis and treatment of impaired wound healing in diabetes mellitus: new insights. Adv Ther 2014;31(8):817-36.

[14] Mehta S, Watson JT. Platelet rich concentrate: basic science and current clinical applications. J Orthop Trauma 2008;22(6):432-8.

[15] Singer AJ, Clark RA. Cutaneous wound healing. N Engl J Med 1999;341(10):738-46.

[16] Knighton DR, Ciresi K, Fiegel VD, et al. Stimulation of repair in chronic, nonhealing, cutaneous ulcers using platelet-derived wound healing formula. Surg Gynecol Obstet 1990;170(1):56-60.

[17] Bielecki TM, Gazdzik TS, Arendt J, et al. Antibacterial effect of autologous platelet gel enriched with growth factors and other active substances: an in vitro study. J Bone Joint Surg 2007;89(3):417-20.

[18] Park KY, Kim IS, Yeo IK, et al. Treatment of refractory venous stasis ulcers with autologous platelet-rich plasma and light-emitting diodes: a pilot study. J Dermatolog Treat 2013;24(5):332-5.

[19] Nikolidakis D, Jansen JA. The biology of platelet-rich plasma and its application in oral surgery: literature review. Tissue Eng Part B Rev 2008;14(3):249-58.

Davis VL, Abukabda AB, Radio NM, et al. Platelet-rich preparations to improve healing. Part I: workable options for every size practice. J Oral Implantol 2014;40(4):50010. 